\title{
High incidence of endogenous depression in migraine: confirmation by tyramine test
}

\author{
Joan Jarman, Margaret Fernandez, P T G Davies, Vivette Glover, T J Steiner, \\ C Thompson, F Clifford Rose, $M$ Sandler
}

\begin{abstract}
Forty patients with migraine who were attending a specialist clinic were interviewed with the Schedule for Affective Disorders and Schizophrenia - Lifetime version. Sixteen $(40 \%)$ had a history of major depression which was of endogenous type in 15, according to Research Diagnostic Criteria. The tyramine test, a previously established trait marker for endogenous depression, showed that the migraine group as a whole had significantly low values compared with 14 normal controls, due almost entirely to low values in the endogenous depressive subgroup; there were no differences between diet-sensitive and non-dietsensitive migraine patients. Thus depression in patients with migraine seems unlikely to be secondary to migraine per se. A substantial subgroup of patients with migraine may possess an inherent predisposition to endogenous depression.
\end{abstract}

It has often been suggested that migraine is linked to personality traits such as obsessionality, over-conscientiousness, rigidity and excessive drive, ${ }^{12}$ but these associations are uncertain and controversial. ${ }^{3}$ Connections between depression and migraine are better established. An increased prevalence of depression among migraine patients, ${ }^{45}$ as well as a raised incidence of migraine among patients receiving treatment for depression ${ }^{6}$ have been reported. There is evidence to suggest that this association is not solely a hospital outpatient clinic phenomenon; two studies ${ }^{37}$ have shown that depression, and also anxiety, are migraine accompaniments in the general population. Furthermore, an association between major depression and migraine has recently been shown in subjects selected at random from a community sample. ${ }^{6}$

There may well be biochemical features common to both migraine and depression. There is evidence of a disturbance in 5hydroxytryptaminergic systems in each, ${ }^{89}$ while both respond to tricyclic antidepressants. ${ }^{10} \mathrm{~A}$ further biochemical deficit, recorded in both, is in tyramine sulphoconjugation. In 1971 we and others, observed a deficit in urinary excretion of tyramine-O-sulphate following an oral tyramine load, in a group of psychiatrically unclassified patients with so-called dietary migraine, ${ }^{112}$ although we later had difficulty in replicating this find- ing (unpublished data). Subsequently, we identified a similar sulphoconjugation deficit present as a trait marker in depression ${ }^{13} 14$ which later proved specific for endogenous depression. ${ }^{15}$ Its biochemical basis remains unclear.

In this study we have obtained detailed psychiatric histories from a group of patients attending a specialist headache clinic, and measured tyramine sulphate excretion in them, to clarify whether low tyramine sulphoconjugation is a characteristic of migraine patients in general, or of a depressed subgroup only.

\section{Methods}

From 176 patients seen consecutively in the Princess Margaret Migraine Clinic, Charing Cross Hospital, for treatment of common or classical migraine, diagnosed according to Vahlquist's criteria, ${ }^{17} 40$ agreed to return to participate in the study. All the patients that were approached to take part in the study completed the self-rating Hospital Anxiety and Depression Scale, ${ }^{18}$ which provided a crude estimate of their current anxiety and depression. Control subjects $(n=14)$ not regularly suffering from any form of headache and otherwise healthy, were recruited from laboratory personnel. The mean ages $(\mathrm{SD})$ for controls and patients were $34 \cdot 2(11 \cdot 8)$ years and $39.9(10.6)$ years, respectively. The patient group comprised eight males and 32 females and the controls four males and 10 females.

In the 48 hours preceding oral tyramine challenge, patients and controls refrained from paracetamol or ascorbic acid ingestion and followed a low tyramine diet. They fasted overnight with only water to drink and, at the start of the test, emptied their bladders and swallowed a capsule containing $125 \mathrm{mg}$ tyramine hydrochloride. All urine was then collected for exactly 3 hours and its volume determined. An aliquot was stored frozen at $-20^{\circ} \mathrm{C}$ before measurement of tyramine-Osulphate by a gas chromatographic method. ${ }^{19}$ Although patients were asked to refrain from paracetamol in the 48 hours before the oral tyramine challenge, a number of them had taken medication in the previous week (table 1). Only two patients were taking antidepressants, and both of these were diagnosed as having a history of endogenous depression.

During the three hour period of the test, patients were interviewed by a trained clinical rater (MF), using the Schedule for Affective Disorders and Schizophrenia Lifetime Version 
Table 1 Drug intake by patients in the week preceding oral tyramine challenge

\begin{tabular}{|c|c|c|}
\hline Drug & $n$ & $\begin{array}{l}\text { Tyramine-O-sulphate } \\
\text { excretion }(\mathrm{mg} / 3 \mathrm{~h}) \\
\text { median (range) }\end{array}$ \\
\hline $\begin{array}{l}\text { Drug-free } \\
\text { Propranolol/atenolol } \\
\text { Non-steroidal anti- } \\
\text { inflammatory drugs }\end{array}$ & $\begin{array}{r}11 \\
18 \\
9\end{array}$ & $\begin{array}{l}4 \cdot 4(2 \cdot 0-9 \cdot 1) \\
4 \cdot 4(1 \cdot 9-8 \cdot 1) \\
4 \cdot 5(2 \cdot 2-8 \cdot 1)\end{array}$ \\
\hline $\begin{array}{l}\text { Otherst } \\
\text { Pizotifen } \\
\text { Antidepressants }\end{array}$ & $\begin{array}{l}8 \\
3 \\
2\end{array}$ & $\begin{array}{l}4 \cdot 6(2 \cdot 6-6 \cdot 2) \\
4 \cdot 7(2 \cdot 2-6 \cdot 2) \\
3 \cdot 1(3 \cdot 8-4 \cdot 4)\end{array}$ \\
\hline $\begin{array}{l}\text { Drug-free patients only } \\
\text { History of endogenous } \\
\text { depression }\end{array}$ & 5 & $2.9(2.0-3.4)^{\star}$ \\
\hline $\begin{array}{l}\text { No history of endogenous } \\
\text { depression }\end{array}$ & 6 & $5 \cdot 1(4 \cdot 3-9 \cdot 1)$ \\
\hline
\end{tabular}

(SADS-L) $)^{20}$ to assess current and previous incidence of psychiatric disorder. Endogenous depression was diagnosed according to Research Diagnostic Criteria. ${ }^{21}$

\section{Results}

Prevalence of psychiatric disorder

To assess whether patients who had volunteered to have the oral tyramine test plus psychiatric interview were representative of the clinic population, anxiety and depression scores on the Hospital Anxiety and Depression Scale ${ }^{18}$ for the 40 participants and the 136 nonparticipants were compared. Scores greater than 11 indicated levels of anxiety or depression consistent with the presence of a clinical mood disorder. On the anxiety scale, scores $>11$ were obtained by $20 \%$ of the patients volunteering compared with $38 \%$ of non volunteers, and on the depression scale, by $8 \%$ of volunteers compared with $10 \%$ of non volunteers. Therefore, the patients participating in the study actually appeared to be less anxious and depressed than the rest of the patients attending the clinic

Among the 40 volunteers, a lifetime history of major depression was by far the most prevalent psychiatric disorder (table 2), present in $40 \%$ of patients, with $15 \%$ being clinically depressed at the time of examination. All but one of the $40 \%$ also fulfilled Research Diagnostic Criteria for endogenous depression. This incidence of depression is in excess of literature estimates of lifetime occurrence of major depression in the general population (8-12\% for males; $20-25 \%$ for females). ${ }^{22}$

Tyramine sulphoconjugation

Mean urinary three hour tyramine-O-sulphate output was significantly lower in the 40 migraine patients compared with controls [ 4.69 $(1.74) \mathrm{mg} / 3$ hour and $6 \cdot 12(1.67) \mathrm{mg} / 3$ hour; $\mathrm{p}$ $<0.02]$. When the migraine patients were divided into those with a history of endogenous depression and those without, the former showed a significantly lower three hour excretion than the latter $(p<0.02)$ and the control group ( $p<0.001)$. Those patients without a history of endogenous depression had excretion values not significantly different from control values (fig). Our earlier studies ${ }^{12}$
Table 2 Major psychiatric disorders in 40 migraine patients diagnosed using the $S A D S-L$

\begin{tabular}{lll}
\hline & $\begin{array}{l}\text { Lifetime } \\
\text { incidence } \\
n\end{array}$ & $\begin{array}{l}\text { Present } \\
\text { state } \\
n\end{array}$ \\
\hline Major depression & 16 & 6 \\
$\quad$ (Endogenous depression) & $(15)$ & $(6)$ \\
Minor depression & 2 & 1 \\
Alcoholism & 1 & 1 \\
Labile personality & 1 & 1 \\
Antisocial personality & 1 & 1 \\
Generalised anxiety disorder & 1 & 0 \\
Agoraphobia & 1 & 0 \\
No psychiatric disorder & 17 & 30 \\
\hline
\end{tabular}

showed excretion levels for endogenous depressives to fall at or below $4.1 \mathrm{mg} / 3$ hour, which was therefore proposed as the diagnostic cut-off point for the disorder. Our present data show that $73 \%$ of the endogenous depressives, as opposed to $12 \%$ of the non-depressive migraine group, fell into this "low conjugator" category, a highly significant difference ( $\mathrm{p}<$ 0.0003, Fisher's Exact Test, two-tailed).

A number of patients had taken medication in the week preceding oral tyramine challenge. To check that these forms of medication were not responsible for the differences in tyramine conjugation noted between patients with and without a history of endogenous depression, output values in patients who had taken different categories of drugs were compared (table 1). No significant differences between patients taking any type of drug and drug-free patients were observed and the significant difference in conjugated-tyramine output between depressed and non-depressed groups persisted even when only drug-free patients were considered.

When patients were classified as dietsensitive, that is, those who observed improvement in their migraine attacks when avoiding supposedly responsible foods and deterioration when they were reintroduced, and non-diet-sensitive, no differences in tyramine sulphoconjugation were observed between the two groups, whether or not the endogenous depressives were included in the analysis. Three of the diet-sensitive patients believed that cheese specifically would initiate their attacks; we therefore scrutinised them

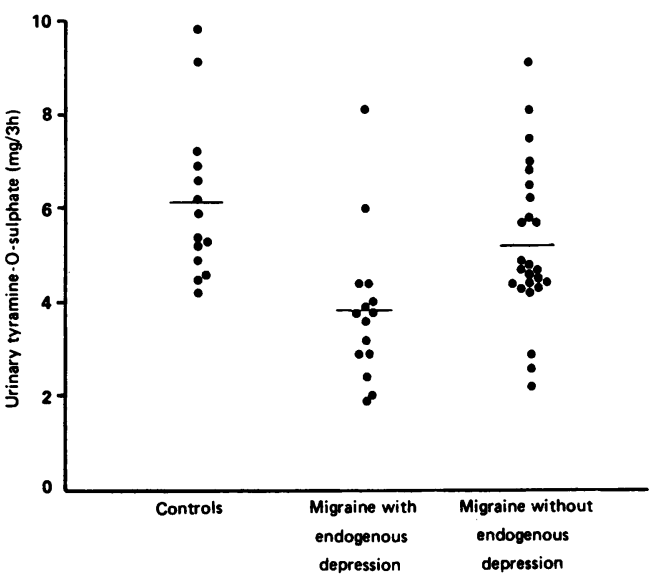

Figure Urinary tyramine-O-sulphate excretion in 14 controls (CON), 15 migraine patients with a history of endogenous depression $(M+D)$ and 25 migraine patients with no history of endogenous depression (M). Controls versus $M+D, p<0.001$; versus $M+D, p<0.02$; Controls versus $M$, not significant; Student's $t$ test, twotailed. 
separately but were unable to demonstrate any difference in sulphoconjugation in these patients compared with the control group.

\section{Discussion}

This study demonstrates a high lifetime incidence of endogenous depression in migraine patients, a finding confirmed by the tyramine test, a sensitive biochemical trait marker. ${ }^{15}$ In any investigation of patients attending a specialist clinic, the question arises as to whether the patients, to some extent self-selected, are representative of those in the community. In this study there was some evidence from a self-rating Hospital Anxiety and Depression Scale ${ }^{18}$ to suggest that the patients volunteering for further psychiatric and biochemical evaluation were actually somewhat less anxious and depressed than the non-volunteers. Although not investigated here, it would be of interest to extend these findings by comparing other aspects of personality and also of migraine severity in volunteers and non-volunteers. An overall link between migraine and depression has previously been noted in community studies, ${ }^{6}$ suggesting that the findings in our volunteers may have a wider validity.

A striking and unexpected characteristic of the depressive illness observed in the migraine volunteers was its almost completely endogenous nature, confirmed by tyramine test. Low tyramine conjugation is a trait marker for the endogenous variant of the disorder and is thus independent of present state; it manifests in approximately half the first degree relatives of endogenous depressives, whether or not they have yet experienced an episode of the illness. ${ }^{15}$ It therefore appears likely that the conjugation deficit represents an inborn error of metabolism which can be employed to pinpoint objectively those individuals with a predisposition towards this particular form of depressive illness. The presence of the trait in this group seems to rule out that depression was a secondary reaction to migraine, as had previously been suggested. ${ }^{6}$ It appears more likely that an individual may have a predisposition to both migraine and endogenous depression, which have their basis in a shared set of biochemical abnormalities.

Recently, Hale et al ${ }^{3}$ have shown that possession of the tyramine conjugation deficit by depressives is a predictor for a favourable response to tricyclic antidepressant therapy. It will be interesting to see whether it is similarly a predictor of tricyclic treatment response in migraine.

The sulphoconjugation of tyramine is catalysed by phenolsulphotransferase (PST). Although activity of PST has previously been found to be deficient in the platelets of dietary migraine patients, ${ }^{24} 25$ we have found no significant correlation between either platelet PST $M$ or PST $P$ and urinary excretion of tyramine-O-sulphate in subjects participating in this study (Jarman et al in preparation). This confirms previous work in which no differences were found in platelet PST $M$ or $P$ activity in depressed patients of a type characterised by low excretion of tyramine-O-sulphate compared with controls. ${ }^{26}$
Despite earlier indications to the contrary, ${ }^{11} 12$ the present investigation has failed to demonstrate any correlation between diet sensitivity per se and the tyramine sulphoconjugation deficit. It is only when a lifetime history of endogenous depression is present in the same individual that the conjugation deficit is observed. The original patients with dietary migraine and tyramine conjugation deficiency $^{1112}$ were psychiatrically unclassified. In the light of our findings, it seems likely that the group investigated had included a significant number of patients with the endogenous depression trait.

Joan Jarman and P T G Davies were supported by The Migraine Trust.

1 Wolff HG. Personality features and reactions of subjects with migraine. Arch Neurol Psychiat 1937;37:895-921. 2 Henryk-Gutt R, Rees WL. Psychological aspects of migraine. J Psychosom Res 1973;17:141-53.

3 Crisp AH, Kalucy RS, McGuinness B, Ralph PC, Harris G. Some clinical, social and psychological characteristics of migraine subjects in the general population. Postgrad Med $J$ 1977;53:691-7.

4 Kashiwagi T, McClure JN, Wetzel RD. Headache and psychiatric disorder. Dis Nerv Syst 1972;33:659-63.

5 Kudrow L. Current aspects of migraine headache. Psychosomatics 1978;19:48-57.

6 Merikangas KR, Risch NJ, Merikangas JR, Weissman MM, Kidd KK. Migraine and depression: association and Kidd KK. Migraine and depression: association and
familial transmission. J Psychiat Res 1988;22:119-29.

7 Paulin JM, Waal-Manning HJ, Simpson FO, Knight RG. The prevalence of headache in a small New Zealand town. Headache 1985;25:145-51.

8 Coppen A, Swade C, Wood K, Carroll JD. Platelet 5hydroxytryptamine accumulation and migraine. Lancet 1979;i1:914.

9 Coppen A, Swade C, Wood K. Platelet 5-hydroxytryptamine accumulation in depressive illness. Clin Chim Acta 1978;87:165-8.

10 Couch JR, Ziegler DK, Hassanein RS. Amitriptyline in the prophylaxis of migraine. Neurology 1976;26:121-7.

11 Youdim MBH, Bonham Carter S, Sandler M, Hanington E, Wilkinson $M$. Conjugation defect in diet sensitive migraine. Nature 1971;230:127-8.

12 Smith A, Kellow AH, Mullen PE, Hanington E. Dietary migraine and tyramine metabolism. Nature 1980;230: migrai

13 Sandler M, Bonham Carter S, Cuthbert MF, Pare CMB. Is there an increase in platelet monoamine oxidase activity in depressive illness? Lancet 1975;i:1045-9.

14 Bonham Carter S, Sandler M, Goodwin BL, Sepping P, Bridges PK. Decreased urinary output of tyramine and its metabolites in depression. Br J Psychiat 1978;132:125-32.

15 Hale AS, Walker PL, Bridges PK, Sandler M. Tyramine conjugation deficit as a trait marker in endogenous depressive illness. J Psychiat Res 1986;20:251-61.

16 Harrison WM, Cooper TB, Stewart JW, et al. The tyramine challenge test as a marker for melancholia. Arch Gen Psychiat 1984;41:681-5.

17 Vahlquist B. Migraine in children. Int Arch Allergy 1955;7:348-55.

18 Zigmond AS, Snaith RP. Hospital anxiety and depression scale. Acta Psychiat Scand 1983;67:361-70.

19 Walker PL, Sandler M. The analysis of urinary meta- and para-tyramine by gas chromatography with electron capture detection. Ann Clin Biochem 1988;25:304-9.

20 Spitzer RL, Endicott J. Schedule for affective disorders and schizophrenia-lifetime version. New York: New York Biometrics Research Division, New York State Psychiatric Institute, 1975.

21 Spitzer RL, Endicott J, Robins E. Research diagnostic criteria for a selected group of functional disorders, ed 3. New York: Biometrics Research Division, New York State Psychiatric Institute, 1977.

22 Charney EA, Weissman MM. Epidemiology of depressive and manic syndromes. In: A Georgotas, $R$ Cancro, eds. Depression and Mania. Amsterdam: Elsevier, 26-52.

23 Hale AS, Sandler M, Hannah P, Bridges PK. Tyramine conjugation test for prediction of treatment response in conjugation test for prediction of treatm

24 Littlewood J, Glover V, Sandler M, Petty R, Peatfield R, Rose FC. Platelet phenolsulphotransferase deficiency in Rose FC. Platelet phenolsulphotransf

25 Soliman H, Pradalier A, Launay J-M, Dry J, Dreux C. Decreased phenol and tyramine conjugation by platelets in dietary migraine. In: F C Rose, ed. Advances in Headache Research. London: John Libbey, 117-21.

26 Bonham Carter SM, Glover V, Sandler M, Gillman PK, Bridges PK. Human platelet phenolsulphotransferase: separate control of the two forms and activity range in depressive illness. Clinica Chimica Acta 1981;117: 333-44. 